\title{
CALCUL HYDRAULIQUE \\ DES CANAUX D'ASSAINISSEMENT \\ AGRICOLE
}

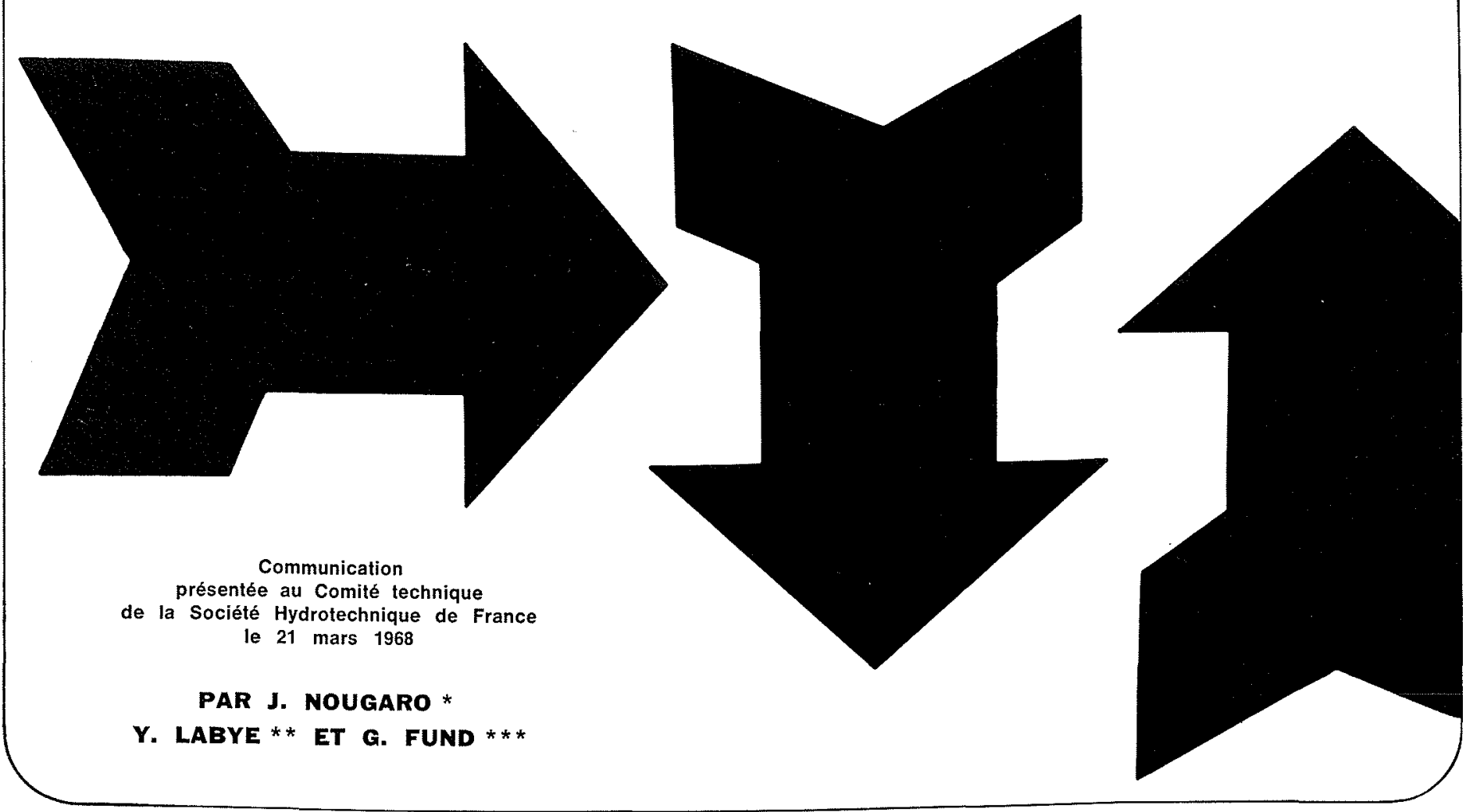

\section{I. - Introduction}

La présente note fait suite à une communication que nous avons présentée dans le cadre du XII" Congrès de l'A.I.R.H. tenu à Fort-Collins en septembre 1967. Notre but est essentiellement utilitaire : c'est la raison pour laquelle la présente note ne comporte pas de démonstrations détaillées.

Les formules, tables et abaques présentés ci-dessous ont été établis afin de déterminer les dimensions des canaux dans les projets d'assainissements agricoles.

Ceci nous conduira à insister tout particulièrement sur le cas des canaux à profil en long horizontal (canaux dits à fond plat).

\section{II. - Notations utilisées}

Les profils en travers et en long sont présentés par les figures 1 et $2 ; A^{\prime} B^{\prime}$ et $A B$ sont horizontales.

Professeur, Lauréat de l'Institut, Directeur Technique de l'Institut de Mécanique des Fluides de Toulouse (France).

* Docteur ès-Sciences Physiques, Ingénieur du Génie Rural et des Eaux et Forets, Paris (France)

** Ingénieur de l'E.N.S.A. Remes, Ingénieur de la Section Spéciale l'Hydraulique de 1'E.N.S.E.E.H., Toulouse (France).
$S$ est l'intersection des droites $\mathrm{AA}^{\prime}$ et $\mathrm{BB}^{\prime}$. La courbe AMB peut être quelconque; toutefois si l'on veut poursuivre le calcul jusqu'à un niveau de la surface libre $\mathrm{A}^{\prime \prime} \mathrm{B}^{\prime \prime}$, les courbes $\mathrm{AA}^{\prime \prime}$ et $\mathrm{BB}^{\prime \prime}$ doivent être polygonales.

$Q$ : débit du canal; $\Omega$ : section mouillée; $X$ : périmètre mouillé; $\Omega_{0}$ : aire de la section délimitée par $\mathrm{AB}$ et l'arc $\mathrm{A} \widehat{\mathrm{MB}} ; \mathrm{X}_{0}$ : longueur de larc $\mathrm{A} \widehat{\mathrm{MB}} ; \boldsymbol{i}$ : pente du profil en long ( $i$ algébrique); $\beta_{1}$ et $\beta_{2}$ : angle des berges avec $\mathrm{AB} ; l=\mid \mathrm{AB}$.

On pose :

$$
\begin{gathered}
\operatorname{tg} \beta_{1}=\frac{1}{s_{1}}, \quad \operatorname{tg} \beta_{2}=\frac{1}{s_{2}} \\
\sin \beta_{1}=\frac{1}{t_{1}}, \quad \sin \beta_{2}=\frac{1}{t_{2}} \\
t=\frac{t_{1}+t_{2}}{2}, \quad s=\frac{s_{1}+s_{2}}{2}, \quad m=\frac{2 t}{s}
\end{gathered}
$$

- pour $\beta_{1}$ et $\beta_{2}$ inférieurs à $\pi / 2$ on a :

$$
m>2
$$

$-\operatorname{pour} \beta_{1}=\beta_{2}=\beta<(\pi / 2)$ on $\mathrm{a}$ :

$$
\begin{gathered}
s=\frac{1}{\operatorname{tg} \beta}, \quad t=\frac{1}{\sin \beta}, \quad m=\frac{2 t}{s}=\frac{2}{\cos \beta}>2 \\
\text { et } 1+s^{2}=t^{2}
\end{gathered}
$$

Dans tous les cas, on a :

$$
\Omega=\operatorname{sh}^{2}+l h+\Omega_{0} ; \quad \mathrm{X}=\mathrm{X}_{0}+2 \text { th }
$$




\section{III. - Principe du procédé}

1. Equation de base.

avec :

$$
\frac{d \alpha \mathrm{V}^{2}}{2 g}+d h=(\mathrm{J}-i) d x
$$

$$
\begin{gathered}
J=\frac{\mathrm{V}^{2}}{\mathrm{CR}^{4 / 3}}, \quad \mathrm{~V}=\frac{\mathrm{Q}}{\Omega}, \quad \mathrm{R}=\frac{\Omega}{\mathrm{X}}, \quad \mathrm{C}=\frac{1}{n^{2}} \\
\text { (Manning Strickler); } \\
\alpha=1+\frac{\mathrm{K}}{\mathrm{CR}^{1 / 3}} \text { (Bazin) }
\end{gathered}
$$

Afin de simplifier les formules, nous adopterons ici $\alpha=1$, ce qui évite l'intervention d'un terme complémentaire $\mathrm{K}_{3} f_{3}(u)$ dans l'équation (3) ci-dessous. Cette simplification est justifiée ici du fait que $\alpha$ varie peu et que dans les canaux d'assainissement $V$ est faible, en général. La consultation des tables montre d'ailleurs que $d \alpha \mathrm{V}^{2} / 2 g$ est faible devant $d h$ dans la plupart des cas.

On a done :

$$
-\frac{Q^{2}}{g \Omega^{3}} d \Omega+d h=\left[\frac{\mathrm{Q}^{2} \mathrm{X}^{4 / 3}}{\mathrm{C} \Omega^{10 / 3}}-i\right] d x
$$

\section{Formules utilisées.}

On déduit immédiatement de (2) la formule générale :

où :

$$
\frac{\mathrm{K}_{1}-f_{1}(u)}{f_{2}(u)-\mathrm{K}_{2}} d u=\mathrm{K}_{3} d x
$$

$\mathbf{K}_{1}=\frac{g \theta^{3} l^{\prime 2}}{s^{\prime 3} \mathrm{Q}^{2}}, \quad \mathrm{~K}_{2}=\frac{i \mathrm{C}^{\prime} \theta^{2} l^{\prime 10 / 3}}{s^{\prime 10 / 3} \mathrm{Q}^{2}}, \quad \mathrm{~K}_{3}=\frac{g s^{\prime 4 / 3}}{\mathrm{C}^{\prime} l^{\prime 4 / 3}}$

$\theta, u, f_{1}(u), f_{2}(u), l^{\prime}, s^{\prime}, \mathrm{C}^{\prime}$ étant obtenus de la façon suivante :

A. $s\left(\Omega_{0}^{2}+l^{2}\right) \neq 0$.

$s^{\prime}=s, \quad \theta=l^{\prime}=l^{(1-|\varepsilon|)} \sqrt{ }\left|4 s \Omega_{0}-l^{2}\right|+(1-[\varepsilon \mid)$,

$\mathrm{C}^{\prime}=\mathrm{C} \lambda^{\prime 4 / 3}$

où $\varepsilon$ est égal à $+1,-1$, ou 0 selon que $\left(4 s \Omega_{0}-\ldots l^{2}\right)$ est positif, négatif ou nul.

$$
\begin{gathered}
\lambda^{\prime}=\frac{s l^{\prime}}{s \mathrm{X}_{0}+t\left(l^{\prime}-l\right)}, u=\frac{2 \mathrm{sh}+l}{2 l^{\prime}}-\frac{1}{2}, \\
m^{\prime}=m \lambda^{\prime}=\frac{2 t}{s} \lambda^{\prime} \\
f_{1}(u)=\frac{1+2 u}{\left[\left(u+\frac{1}{2}\right)^{2}+\frac{\varepsilon}{4}\right]^{3}} \\
f_{2}(u)=\frac{\left[1+m^{\prime} u\right]^{4 / 3}}{\left[\left(u+\frac{1}{2}\right)^{2}+\frac{\varepsilon}{4}\right]^{10 / 3}}
\end{gathered}
$$

On a aussi :

$$
\begin{gathered}
\Omega=\frac{l^{\prime 2}}{s}\left[\left(u+\frac{1}{2}\right)^{2}+\frac{\varepsilon}{4}\right] \quad \mathrm{X}=\frac{l^{\prime}}{\lambda^{\prime}}\left[1+m^{\prime} u\right] \\
h=\frac{l^{\prime}}{s} u+\frac{l-l^{\prime}}{s}
\end{gathered}
$$

Pour les sections trapézoïdales évasées vers le haut, on a :

$$
\begin{gathered}
\theta=l^{\prime}=l, \quad \lambda^{\prime}=1, \quad m^{\prime}=m=\frac{2 t}{s}>2 \\
\varepsilon=-1, \quad u=\frac{s h}{l} \\
\mathrm{~K}_{1}=\frac{g l^{5}}{s^{3} \mathrm{Q}^{2}}, \quad \mathrm{~K}_{2}=\frac{i \mathrm{C} l^{16 / 3}}{s^{10 / 3} \mathrm{Q}^{2}}, \quad \mathrm{~K}_{3}=\frac{g s^{4 / 3}}{\mathrm{C} l^{4 / 3}} \\
\Omega=\frac{l^{2}}{s}\left(u^{2}+u\right)=\frac{l^{2}}{s} \Omega^{\star}(u), \mathrm{X}=l(1+m u)
\end{gathered}
$$

B. $s=0$.

On a :

$$
\begin{gathered}
s^{\prime}=t, \quad \mathrm{C}^{\prime}=\mathrm{C}, \quad l^{\prime}=l, \quad \theta=\frac{\mathrm{X}_{0} l-2 t \Omega_{0}}{l}, \\
u=\frac{\left[l h+\Omega_{0}\right] t}{\theta l} \\
\Omega=\frac{\theta l u}{t}, \quad \mathrm{X}=\theta(1+2 u), \quad h=\frac{\theta u}{t}-\frac{\Omega_{0}}{l} \\
f_{1}(u)=\frac{1}{u^{3}}, \quad f_{2}(u)=\frac{(1+2 u)^{4 / 3}}{u^{10 / 3}}
\end{gathered}
$$

Pour :

on a :

$$
\beta_{1}=\beta_{2}=\frac{\pi}{2}
$$

$$
\left.s^{\prime}=t=1 \text { (fig. } 3\right)
$$

Pour une section rectangulaire, on a :

$$
\begin{aligned}
& \Omega_{0}=0, \quad \mathrm{X}_{0}=l=\theta, \quad u=\frac{h}{l} \\
& \Omega=l^{2} u, \quad \mathrm{X}=l(1+2 u)
\end{aligned}
$$

C. $\Omega_{0}=l=0$ (section triangulaire).

$$
\begin{gathered}
s^{\prime}=s, \quad \theta=l^{\prime}=1 \\
u=s h, \quad h=\frac{u}{s}, \quad \Omega=\frac{u^{2}}{s}, \quad \mathrm{X}=\frac{2 t u}{s}, \quad \mathrm{C}^{\prime}=\frac{\mathrm{C}}{(2 t)^{4 / s}} \\
f_{1}(u)=\frac{2}{u^{3}}, \quad f_{2}(u)=\frac{1}{u^{10 / 3}}
\end{gathered}
$$

3. Remarque sur le choix de la variable $u$.

Cette variable permet d'obtenir une formulation unique pour (3) et (4), valable dans les trois cas possibles; pour les profils le plus souvent rencontrés, section rectangulaire, el section trapézoïdale, isocèle, concave vers le haut, $u$ s'exprime très simplement en fonction de $h, l$ et $\beta$.

La variable $u$ permet de limiter le nombre de valeurs $m$ ' à considérer, lors de l'établissement des tables donnant $f_{i}(u)$, le fait que $m^{\prime}$ figure sous la forme $\left(1+m^{\prime} u\right)^{4 / 3}$ justifiant une interpolation linéaire avec des écarts $m^{\prime}$ importants.

C'est la raison pour laquelle nous l'avons adoptée même pour les sections trapézoïdales où la variable $z=h / l$ conduit à des constantes $\mathrm{K}_{i}$, où $s^{\prime}=1$, donc un peu plus simples, car $s$ figure à une puissance élevée dans les fonctions homologues $f_{i}(s, z)$.

Il faut noter une propriété importante des sections polygonales concaves vers le haut:

- ou bien on a $4 s \Omega_{0}-l^{2}=0$, et on est ramené au cas $C$ en prenant pour origine le point $S$; 


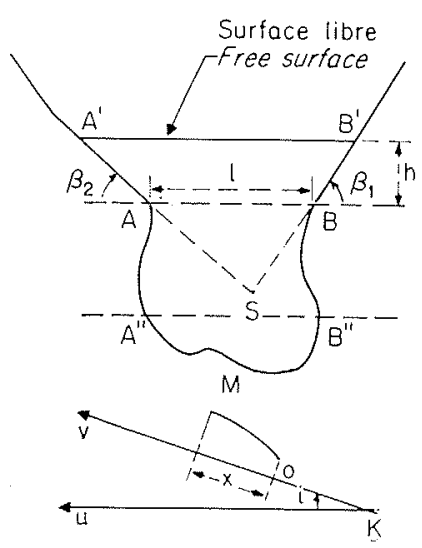

$1 /$ et $2 /$

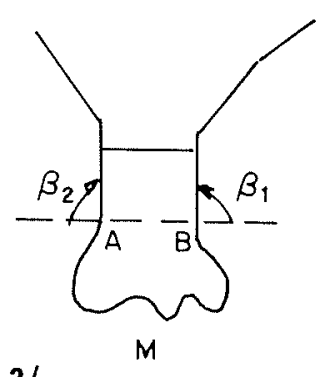

3/

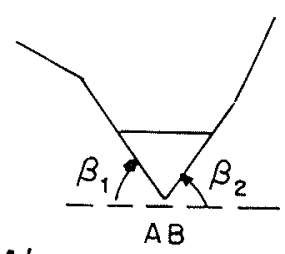

4/

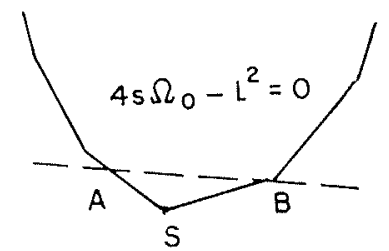

5/

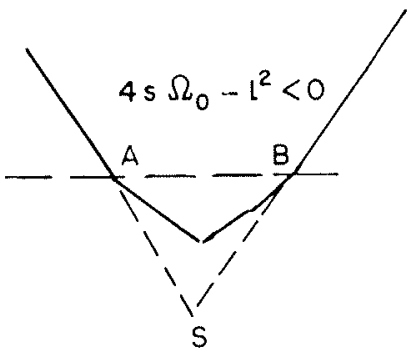

6)
- oll bien on a $4 s \Omega_{0}-l^{2}<0$ et on démontre facilement qu'on a en outre $m^{\prime}>2$.

Il suffit donc que la section polygonale soit concave vers le haut (ce n'est pas une condition nécessaire) pour que les tables relatives aux sections trapézoïdales concaves vers le haut soient applicables.

Négliger $f_{1}(\boldsymbol{u})$ revient à négliger le terme :

$$
d \alpha \mathrm{V}^{2} / 2 g
$$

On démontre facilement que ceci entraine un écart sur $h$ strictement inférieur à $\alpha_{0} V_{0}^{2} / 2 g$, soit de l'ordre de $5 \mathrm{~cm}$ pour un canal en terre où $\mathrm{V}<1 \mathrm{~m} / \mathrm{s}$.

En fait l'écart véritable est nettement plus faible ainsi que le montre une simple consultation des tables donnant $f_{1}(u)$.

La relation (3) devient alors $\left(3^{\star}\right)$ :

$$
\frac{d u}{f_{2}(u)-\mathrm{K}_{2}}=\mathrm{K}_{4} d x
$$

avec : $\quad \mathrm{K}_{\mathbf{4}}=\frac{\mathbf{K}_{3}}{\mathrm{~K}_{1}}=\frac{s^{\prime 13 / 3} \mathrm{Q}^{2}}{\mathrm{C}^{\prime} \theta^{3} l^{\prime 10 / 3}}$

$h_{n}, h_{c}, i_{c}^{\prime}, i_{c}$ désignant respectivement la hauteur normale, la hauteur critique, la pente critique relative $\grave{a} Q$, la pente critique relative à $h$, on obtient en posant $f_{2}(u)=\psi\left(f_{1}(u)\right)$ les formules (5) suivantes, où $u, u_{n}, u_{c}$ sont les valeurs de $u$ relatives à $h, h_{n}, h_{c}$ :

$$
\begin{aligned}
& f_{2}\left(u_{n}\right)=\mathrm{K}_{2} \\
& f_{1}\left(u_{c}\right)=\mathbf{K}_{1} \\
& i^{\prime}{ }_{c}=\frac{\psi\left(\mathrm{K}_{1}\right)}{\mathrm{K}_{2}{ }^{*}} \\
& i_{c}=\frac{f_{2}(u)}{\mathrm{K}_{2}{ }^{*}}
\end{aligned}
$$

avec :

Si en outre $i \neq 0$ on a :

$$
\mathrm{K}_{2}{ }^{*}=\mathrm{K}_{2}(i==1)=\frac{\mathrm{C}^{\prime} \theta^{2} 10 / 3}{s^{10 / 3} \mathrm{Q}^{2}}
$$

$$
i_{c}^{\prime}=\frac{i \Psi\left(\mathrm{K}_{1}\right)}{\mathbf{K}_{2}} \quad i_{c}=\frac{i f_{2}(u)}{\mathrm{K}_{2}}=\frac{i f_{2}(u)}{f_{2}\left(u_{n}\right)}
$$

Pour $i=0$ on a $f_{2}\left(u_{n}\right)=0, h_{n}=\infty$.

On remarque que $(5,1)$ permet de calculer les canaux en régime uniforme, et que (3) ou $\left(3^{*}\right)$ permettent de calculer soit les canaux d'assainisse- ment où $f_{2}(u)>K_{2}$ puisque $i$ est faible, soit les courbes de remous habituelles où $f_{2}(\boldsymbol{u})<\mathbf{K}_{2}$.

Lorsque $i=0$, la formule (3) devient :

ou :

$$
\left|\frac{1}{\mathbf{K}_{4}} \mathbf{F}(u)-\frac{1}{K_{3}} \mathbf{G}(u)\right|_{u_{\mathrm{u}}}^{u_{1}}=x_{1}-x_{0}
$$

$\left|\mathrm{F}(\boldsymbol{u})-\mathrm{K}_{5} \mathrm{G}(\boldsymbol{u})\right|_{u_{0}}^{u_{1}}=\int_{x_{0}}^{x_{1}} \mathrm{~K}_{4} d x=|\mathbf{I}(u)|_{u_{0}}^{u_{1}}$ avec :

$$
\begin{aligned}
& \mathrm{K}_{5}=\frac{1}{\mathrm{~K}_{1}}=\frac{s^{\prime 3} \mathrm{Q}^{2}}{g \theta^{3} l^{\prime 2}} \\
& \mathrm{~F}(u)=\int_{0}^{u} \frac{1}{f_{2}(u)} d u \\
& \mathrm{G}(u)=\int_{0}^{u} \frac{f_{1}(u)}{f_{2}(u)} d u
\end{aligned}
$$

On utilisera aussi :

homologue de $\left(3^{*}\right)$.

$$
|\mathbf{F}(u)|_{u_{i t}}^{u_{1}}=\int_{x_{01}}^{x_{1}} \mathbf{K}_{4} d x
$$

Posons :

$$
\mu(u)=\frac{\mathbf{K}_{2}}{f_{2}(u)}\left[\frac{\mathbf{K}_{2}}{f_{2}(u)}-\frac{f_{1}(u)}{\mathbf{K}_{1}}\right]
$$

$\mu$ (u) est une fonction croissante de $u$ éventuellement négative.

On a :

$$
\begin{aligned}
\frac{\mathrm{K}_{1}-f_{1}(u)}{f_{2}(u)-\mathrm{K}_{2}} & =[1+\mu(u)] \mathrm{K}_{1} \\
& \times\left[\frac{1}{f_{2}(u)}-\mathrm{K}_{5} \frac{f_{1}(u)}{f_{2}(u)}+\mathrm{K}_{2} \frac{1}{\left[f_{2}(u)\right]^{2}}\right]
\end{aligned}
$$

II résulte que la substitution de (7) ci-dessous à (3) entraine une erreur relative $\nu$ sur le premier membre dont le signe est fixé si :

$$
\mu\left(u_{0}\right) \mu\left(u_{1}\right)>0
$$

et dans tous les cas on a :

$$
\nu<\sup \left[\left|\mu\left(u_{0}\right)\right|,\left|\mu\left(u_{1}\right)\right|\right]
$$

$$
\mathbf{F}(u)-\mathbf{K}_{5} \mathbf{G}(u)+\left.\mathbf{K}_{2} \mathbf{R}(u)\right|_{u_{0}} ^{u_{1}}=\int_{x_{0}}^{x_{1}} K_{4} d x
$$




$$
R(u)=\int_{0}^{u} \frac{1 \cdot d u}{\left[f_{2}(u)\right]^{2}}
$$

Non seulement la formule (7) donne, ainsi que le montrent les tables, des résultats précis mème pour des angles $i$ assez grands, mais on peut définir ce qu'on entend par canal à faible pente. C'est un canal où l'on a $\nu<p$, $p$ étant une erreur fixée a priori.

Notons que pour les canaux trapézoïdaux on a :

$$
\begin{gathered}
\mathrm{K}_{4}=\frac{s^{13 / 3} \mathrm{Q}^{2}}{\mathrm{C} l^{19 / 3}}, \quad \mathrm{~K}_{5}=\frac{s^{3} \mathrm{Q}^{2}}{g^{5}}, \\
\mathrm{~F}(u)=\int_{0}^{u} \frac{\left(u^{2}+u\right)^{10 / 3}}{(1+m u)^{4 / 3}} d u \\
\mathrm{G}(u)=\int_{0}^{u} \frac{(1+2 u)\left(u^{2}+u\right)^{1 / 3}}{\left(1+m^{\prime} u\right)^{1 / 3}} d u \\
\mathrm{R}(u)=\int_{0}^{u} \frac{\left(u^{2}+u\right)^{20 / 3}}{(1+m u)^{8 / 3}} d u
\end{gathered}
$$

\section{IV. - Utilisation pratique des formules}

Les abaques, tables, graphiques figurant en annexe permettent de calculer les constantes $K_{i}$ et donnent les valeurs des fonctions de $u$ on des fonctions de $\left(u, u_{0}\right)$ à utiliser. Ces tables montrent que $G(u)$ varie peu, alors que $F(u)$ est une fonction très croissante de $\boldsymbol{u}$. Ceci, et le fait que $i$ et $V$ sont généralement faibles dans les réseaux d'assainissement ont pour conséquence que la formule $\left(6^{*}\right)$ fournit presque toujours une valeur très approchée de la solution, et de toute manière suffisamment exacte pour déterminer si l'on doit faire appel aux formules plus complexes. L'estimation de $u_{1}$ et $u_{0}$ ainsi fournie est assez bonne pour déterminer si un des deux termes $f_{i}, K_{i}$ est négligeable devant l'autre. De même la valeur du terme «en G(u) » où l'on donne à $u$ la valeur donnée par $\left(6^{*}\right)$ est assez précise pour qu’on ramène $6^{\prime}$ à $6^{*}$ par passage de $G\left(u_{6^{*}}\right)$ au second membre.

En fail les calculs se révèlent très rapides, lorsqu'on ne recherche pas une précision illusoire. Il convient à ce sujet de noter que l'estimation du coefficient de rugosité $C$ qui figure dans $K_{4}$ est souvent imprécise.

Il résulte aussi de la croissance rapide de $\mathrm{F}(u)$ avec $u$, qu'une diminution notable de $u_{0}$ n'entraînera, à partir d'une certaine valeur, qu'une augmentation négligeable du débit. Aussi, à titre indicatif, $F(u / 4)$ est de l'ordre de $\frac{1}{100} \mathrm{~F}(u)$. Faire passer $U_{n}$ de $u_{1} / 4$ à $u_{1} / 8$ fait plus que doubler la vitesse $V_{0}$ pour une augmentation du débit $1 / 200$ dans le cas des sections concaves vers le haut qui seules seront considérées ci-après.

Réciproquement, une légère augmentation de $l$, en laissant $u_{1}$ invariant, permet d'accroître considérablement $u_{0}$ et par suite $1 / \mathrm{V}_{0}$. Il est clair que cette remarque reste valable pour la plupart des sections non concaves susceptibles d'être rencontrées et d'ailleurs fort peu utilisées.
1. Cas $i=0$.

A. - DÉterminer Les dIMENSIONS D'UN CANAL DONT L'IMPLANTATION ET LA DISTRIBUTION DES DÉBITS SONT FIXÉES.

Dans ce paragraphe on se limitera aux sections trapézoïdales, qui, sauf exception, sont adoptées en pareil cas.

a) Le canal est situé entre deux stations de pompage (fig. 7 et 8 ).

Un tel canal est toujours un collecteur important. Ce qu'il reçoit par ruissellement direct est négligeable par rapport au débit qu'il transporte. Ce débit ne varie qu'aux confluents avec d'autres canaux qui sont eux-mèmes presque toujours des ouvrages importants. Par suite il est loisible (si nécessaire) de diviser un tel canal en tronçons $T_{1}, T_{j} \ldots T_{n}$, de Iongueur $\mathrm{L}_{1}, \mathrm{~L}_{j} \ldots \mathrm{L}_{n}$, généralement peu nombreux et tels que pour l'élément $\mathrm{T}_{j}$, les quantités $\mathrm{Q}_{j}, \mathrm{C}_{j}$ et par suite $K_{4 j}$ el $K_{5 j}$ sont constantes.

La revanche étant par hypothèse au moins égale à $\delta$, on prendra $B$ situé à une distance $\delta$ du sol afin de ne pas augmenter inutilement le coût de l'ouvrage.

La cote de A n'est pas imposée, mais la vitesse $V_{0}$ doit être inférieure à $V_{0 M}$, fixée par la nature des parois.

De plus, la nécessité de loger l'appareillage, le souci d'éviter la cavitation à l'entrée des pompes hélice font que $h_{0}$ a une borne inférieure $h_{0}$.

La formule (6) montre que pour $l$ fixé, $u_{1}$ [donc $\left.h_{1}=\left(l u_{1} / s\right)\right]$ est une fonction croissante de $u_{0}$. Par suite le coût de l'ouvrage croît avec $u_{0}$. On a donc intérêt à prendre $u_{0}$ [et par suite $\left.h_{0}=\left(l u_{0} / s\right)\right]$ le plus faible possible.

Par suite on prendra $u_{0}=u_{0, l}, u_{0, l}$ étant la plus grande des deux quantités $a$ et $b$ vérifiant respectivement :

$$
\begin{aligned}
& \Omega^{*}(a)=\frac{s \mathrm{Q}_{0}}{l^{2} \mathrm{~V}_{0 \mathrm{M}}} \quad b=\frac{s \bar{h}_{0}}{l} \\
& \left(a=-\frac{1}{2}+\sqrt{\left.\frac{s \mathrm{Q}_{0}}{c^{2} \mathrm{~V}_{0 \mathrm{M}}}+\frac{1}{4}\right)}\right.
\end{aligned}
$$

La relation $\left(6^{\prime}\right)$ donne $u_{n, l}$.

Le coût $\mathcal{C}\left(u_{n, l}, l\right)$ s'exprime alors en fonction uniquement de $l$, et il est loisible de choisir la valeur de $l$ notée $l^{\star}$ qui convient [selon l'allure de $\mathcal{C}(l)$ on ne choisira pas forcément la valeur rendant minimal $\mathcal{C}(l)]$.

En fait, surtout si Q varie le long du canal, on a intérêt à localiser le minimum de $\mathcal{C}(l)$ en recherchant l'optimum de $\mathcal{C}\left(u^{\star}{ }_{n, l}, l\right), u^{\star}{ }_{n, l}$ étant la solution de $\left(6^{\star}\right)$. Comme, pratiquement, on adopte toujours pour $l$ des valeurs « rondes », on sera ramené à calculer $u_{n}$ pour une ou deux valeurs de $l: l_{1}$ et $l_{2}$ encadrant $l^{\star}$.

En conséquence on procède de la façon suivante:

10 On calcule :

$$
\mathrm{A}_{k}{ }_{k}=\frac{s^{13 / 3} \mathrm{Q}_{l k}^{2}\lfloor k}{\mathrm{C}_{k}}, \quad \mathrm{~A}_{j}=\sum_{k=1}^{k=\mathrm{A}^{\prime}{ }_{k},}, \quad K_{5, k}=\frac{s^{3}}{g} \mathrm{Q}_{k}^{2}
$$

On a :

$\mathbf{F}\left(u_{j}\right)-\mathbf{F}\left(u_{0}\right)$

$$
-\frac{1}{l^{5}}\left\{\sum_{k=1}^{k=K_{i, k}}\left[\mathrm{G}\left(u_{k}\right)-\mathrm{G}\left(u_{k-1}\right)\right]\right\}=\frac{\mathrm{A}_{j}}{l^{19 / 3}}
$$


qui se réduit à :

$$
\mathbf{F}\left(u_{j}\right)-\mathbf{F}\left(u_{0}\right)-\frac{K_{5}}{l^{5}}\left[\mathrm{G}\left(u_{j}\right)-\mathrm{G}\left(u_{0}\right)\right]=\frac{\mathrm{A}_{j}}{l^{19 / 3}}
$$

avec :

$$
\mathrm{A}_{j}=\frac{s^{13 / 2} \mathrm{Q}^{2} x_{j}}{\mathrm{C}}
$$

avec : $K^{1 / 5}=\left(s^{3} Q^{2} / g\right)$ lorsque $Q$ et $C$ ne varient pas avec $x$.

$2^{\circ}$ On utilise les formules $\left(6^{\star} j\right)$ et $\left(6^{\star} n\right)$ ci-dessous :

$$
\begin{aligned}
& \mathrm{F}\left(u^{\star}{ }_{j}, l\right)=\mathrm{F}\left(u_{0}, l\right)+\frac{\mathrm{A}_{j}}{l^{19 / 3}} \\
& \mathrm{~F}\left(u^{\star}{ }_{n}, l\right)=\mathrm{F}\left(u_{i}, l\right)+\frac{\mathrm{A}_{n}}{l^{19 / 3}}
\end{aligned}
$$

$\left(6^{\star} n\right)$ fournit $u^{\star}{ }_{n}, l$ et par suite $\mathrm{C}\left(u^{\star}{ }_{n, l}, l\right)=\mathrm{C}^{\star}(l)$, ce qui permet en faisant varier $l$ de façon monotone de localiser $l^{\star}$ et domne done $l_{1}$ et $l_{2}$. Pour chacune de ces deux valeurs $l_{i}(i=1,2)$ on voit alors si l'on peut considérer que $\left(u{ }^{\star}{ }_{n}, l_{i}\right)$ peut ètre «identifié $\gg \dot{a}\left(u_{n}, l_{i}\right)$ solution de $(6 n)$, ce que montre une consultation immédiate des tables. Dans le cas contraire, ce qui est fort rare, on calcule $\left(u^{*}{ }_{j}, l_{i}\right)$ à l'aide de $\left(6^{*} j\right)$ et on obtient $\left(u_{m}, l_{i}\right)$ par l'équation $\left(6^{\prime}, n\right)$ :

$$
\begin{aligned}
& \mathrm{F}\left(u_{n}, l_{i}\right)=\mathrm{F}\left(u_{0}, l_{i}\right)+\frac{\mathrm{A}_{n}}{l_{i}{ }^{19 / 3}} \\
& +\frac{1}{l_{i}} \sum_{k=1}^{l=K_{\bar{j}, k}^{n}}\left[\mathrm{G}\left(u^{\star}{ }_{k}, l_{i}\right)-\mathrm{G}\left(u^{\star}{ }_{k-1}, l_{i}\right)\right] ;\left(6^{\prime} n\right)
\end{aligned}
$$

ce qui fournit le couple $\left(u_{n}, l^{0}\right)$ à adopter.

Lorsque le coût $\mathcal{C}$ est de la forme $\mathcal{C}=\mathrm{K} \Omega\left(u_{n}\right)$ avec $\mathrm{K}$ constant, l'élimination de $l$ entre :

$$
\mathrm{F}\left(u_{n}\right)=\frac{\mathrm{A}_{n}}{l^{19 / 3}}(8) \quad \text { et } \quad \Omega\left(u_{n}\right)=\frac{l^{2}}{s} \Omega^{\star}\left(u_{n}\right)
$$

donne :

$$
\Omega\left(u_{n}\right)=\mathcal{G}_{y}\left(u_{n}\right) \quad \text { où } \quad \frac{d \mathcal{G}_{\mathcal{L}}}{d u_{n}}<0
$$

Par suite, même avec une autre explicitation de $\mathfrak{C}$ et l'utilisation de $(6)$, il est très fréquent que $l^{\star}$ soit très petit on que $\mathcal{C}$ soit une fonction de décroissante de $u_{n}$ donc croissante de $l$, et la section optimale est triangulaire. En fait ceci conduit à augmenter $\mathrm{H}$, mais il est clair que des considérations techniques conduisent à fixer une borne supérieure pour $\mathrm{H}$ et inférieure pour $l$.

Rappelons à nouveau qu'une très légère augmentation de $l$ on $\mathrm{H}$ permet d'accroître considérablement la capacité de transport d'un canal et de diminuer notablement $V_{0}$. On peut done pour une majoration du con̂t, négligeable, obtenir une très grande sécurité.

b) Le canal débouche dans un exutoire à niveau. fixé (fig. 9).

Ici le point $\mathrm{A}$ est fixé. On utilise les mêmes formules que ci-dessus qui fournissent le coût $\mathcal{C}(l)$ en fonction de $l$.

Il est clair que $l^{\star}$ sera obtenu pour $l$ vérifiant $h_{n}-h_{0}=\mathrm{H}^{\star}$ car pour $l$ fixé et :

$$
h_{n}=h_{0}+\mathscr{H}^{\star} \quad \mathrm{I}\left(u_{0}+s \frac{\mathscr{H}^{\star}}{l}\right)-\mathrm{I}\left(u_{0}\right)
$$

est une fonction croissante de $u_{0}$ et de $\mathcal{J}^{\star}$.
Il arrivera que la relation :

$$
u_{n}-u_{0}=\frac{s \mathrm{H}^{\star}}{l}
$$

ne pourra être vérifiée que pour une valeur de $l$ inférieure au minimum $\vec{l}$ imposé à $l$ et le point $B$ sera au-dessous de $\beta^{\prime}$ dont la distance au sol est égal $\grave{a} \delta$. En effet pour atteindre $B$ avec $l=\breve{l}$ et $\mathcal{H} \mathcal{C}^{\star}=\mathrm{H}^{\star}$ on aurail :

$$
u_{0}\left(\bar{l}, \mathrm{H}^{\star}\right)<u_{0}\left(l^{\star}, \mathrm{H}^{\star}\right)
$$

puisque pour $\mathscr{H}^{\star}$ fixé $u_{0}$ décroìt avec $l$.

On dépasserait donc la vitesse $V_{0 y}$ admissible en $O$. Ceci conduit, si l'on veut conserver $\mathbf{H}=\mathrm{H}^{*}$ à prendre $l>\bar{l}$ et par suite obtenir un coût du canal égal à :

$$
\mathcal{C}>\mathrm{C}\left(u_{0}, \bar{l}, \bar{l}\right)=\mathcal{C}\left[u_{0}, \bar{l}, \mathscr{H C}^{\star}(\bar{l})\right]
$$

Là encore on augmentera la sécurité en augmentant légèrement les dimensions de la section.

On a intérêt, à titre indicatif, à calculer la capacité de transport maximale du canal qu'on peut, comme $\mathrm{G}$ est faible, prendre égale à $\mathrm{Q}_{n}+\Delta$, vérifiant :

$$
\begin{aligned}
& \mathrm{F}\left(\bar{u}_{0}, \bar{l}+s \frac{\mathrm{H}^{*}}{l}\right)-\mathrm{F}\left(\bar{u}_{0}, \bar{l}\right)=\frac{s^{13 / 3} \sum\left(\mathrm{Q}_{k}+\Delta\right)^{2} / \mathrm{Cl}}{\bar{l}^{19 / 3}} \\
& \bar{u}_{0}, \bar{l}=\sup \left\{s \frac{\bar{h}_{0}}{\bar{l}},-\frac{1}{2}+\sqrt{\frac{s\left(\mathrm{Q}_{0}+\Delta\right)}{\bar{l}^{2} \mathrm{~V}_{0 \mathrm{M}}}+\frac{1}{4}}\right\} \\
& =\left(u_{0} \bar{l}\right)_{\mathrm{A}}
\end{aligned}
$$

(9) donne $\left(\bar{u}_{0}, l\right) \Delta$ pour $\Delta$ fixé et l'on fait varier $\Delta$ jusqu'à ce que (8) soit vérifié.

Pour un débit $Q_{n}^{\prime}$ compris entre $Q_{n}$ et $Q_{n}+\Delta$ la solution $\left(u_{0}^{\prime}, \bar{l}\right)$ vérifiant :

$$
\left(u_{0}^{\prime}, l\right)=\sup \left\lfloor s \frac{h_{0}}{l},-\frac{1}{2}+\sqrt{\frac{s Q^{\prime} n}{l V_{0 M}}+\frac{1}{4}}\right\rfloor
$$

donne un coût du projet égal à :

$$
\mathcal{C}\left(Q^{\prime}{ }_{n}\right)=\mathcal{C}\left(u^{\prime}, l\right)
$$

avec $\mathscr{H}^{*}<\mathrm{H}^{*}$ tant que $\mathrm{Q}_{n}^{\prime}<\mathbf{Q}_{n}$.

Il est clair que dans ce paragraphe où $A$ est fixé le coût $\mathfrak{C}(l)$ s'exprime directement à partir de $u_{0}, l$ et $l$ car on a :

$$
\mathrm{H}=\frac{l}{s} u_{0}, l+\mathrm{H}^{\star}+\delta
$$

B. - ETUde D'Un CANal dont le profil en trayers EST FIXÉ.

a) Pour une implantation et une distribution des débits fixés, déterminer $h_{n}$ en fonction de $h_{0}$. Application.

Le calcul ci-dessous est valable même si la section n'est pas concave vers le haut.

On utilise la formule $\left(6^{\prime}\right)$.

Pour une position fixée de la surface libre $A B$ entre $A_{r}, B_{r}$ et $A_{r+1}, B_{r+1}$, la variable $h_{r}$. est fixée et comprise entre 0 et $h^{{ }^{*}}$ correspondant à $A_{r+1}$ et $\mathrm{B}_{r+1}$. Par suite $\mathrm{K}_{4}$ et $\mathrm{K}_{3}$ sont fixés si l'on connaît $\mathrm{Q}$.

Soit $u_{r}=u_{r}{ }^{*}$ pour $h_{r}=0, \quad u_{r}=u_{r}{ }^{*} \quad$ pour $h_{r}=h_{r}{ }^{*}$

Lorsque Q est constant tout le long du canal, 

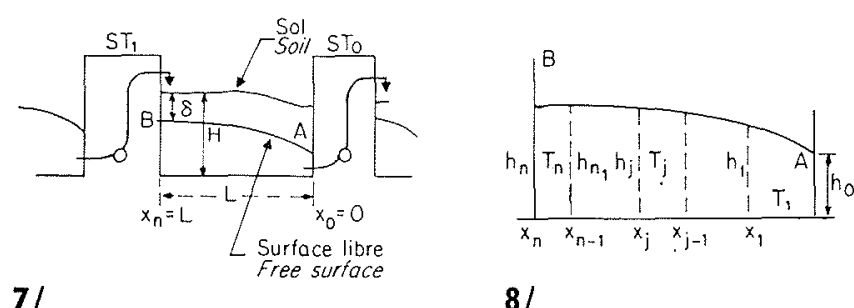

7/

$8 /$

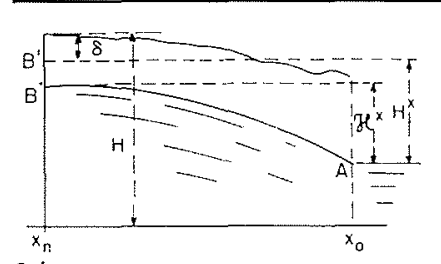

9/

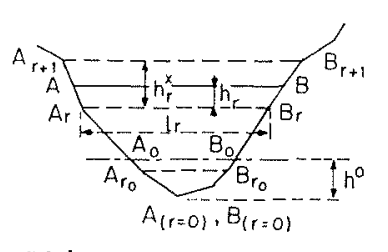

$10 /$

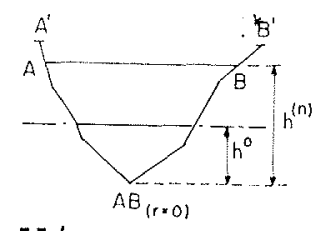

$11 /$

$\mathrm{K}_{3}$ et $\mathrm{K}_{4}$ ne varient qu'avec $r$ et l'on a en appelant $x^{*}{ }_{r}$ l'abscisse correspondant à $\mathrm{A}_{r} \mathrm{~B}_{r}$ :

$$
\begin{gathered}
x-x^{*}{ }_{r}=\mathrm{I}^{{ }_{r}}{ }_{r}\left(u_{r}\right)=\left[\frac{1}{\mathrm{~K}_{4, x}} \mathrm{~F}\left(u_{r}\right)-\frac{1}{\mathrm{~K}_{3, r}} \mathrm{G}\left(u_{r}\right)\right]_{u_{r}{ }^{* *}}^{u_{r}} \\
=\mathrm{I}\left(u_{r}\right)-\mathrm{I}\left(u_{r}{ }^{* *}\right)
\end{gathered}
$$

pour $u_{r}<u^{*}{ }_{r} ; h^{0}$ définit $\Omega_{0}$ et une position $A_{0} B_{0}$ comprise entre deux lignes $\mathrm{A}_{r o} \mathrm{~B}_{r o}$ et $\mathrm{A}_{r o+1} \mathrm{~B}_{r o+1}$.

Par suite, à $h_{0}$ correspond une valeur $u_{00}{ }^{\circ}$.

D'où la relation :

$$
\begin{aligned}
& x-x_{0}=\mathrm{I}_{r o}\left(u^{\star}{ }_{r o}\right)
\end{aligned}
$$

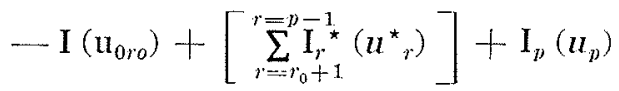

pour $\mathrm{AB}$ compris entre :

$$
\mathrm{A}_{p} \mathrm{~B}_{p} \text { et } \mathrm{A}_{p+1} \mathrm{~B}_{p+1}
$$

L étant la longueur du canal, l'abscisse $x^{(n)}$ de la section amont et $p_{n}$ sont définis par (12) où l'on doit avoir $x^{(n)}-x^{0}=\mathrm{L}$.

On a $x^{\star}{ }_{k}$ en faisant dans (12) :

$$
u_{k}=0 \text { et } p=k
$$

Lorsque $Q$ varie le long du canal, on le divise en tronçon $\mathrm{T}_{j}$ comme dans (A) ci-dessus. $x_{j}$ étant l'abscisse de l'extrémité amont, on utilise (12) pour chaque élément $(j)$ et on substitue dans (12)

$$
x^{(j)} \text { à } x^{(n)} \text { et } x^{(j-1)} \text { à } x^{0}
$$

$h^{(j)}$ joue le rôle de $h^{0}$ pour $h^{(j+1)}$.

Bien entendu il faut que le canal ne déborde pas et $h^{0}$ suffisamment petit.

Le débit aval $Q_{0}$ et la vitesse maximale aval $V_{0 M}$ fixent la section $\Omega_{0 m}$ à laquelle correspond une hauteur $h_{m} ; \bar{h}_{a}$ étant le minimum fixé pour des considérations tenant à l'appareillage, la plus petite valeur de $h^{0}$ est $h^{0}{ }_{*}$ égal à $\sup \left(h^{0}{ }_{m}, h^{0}{ }_{0}\right)$.
Si pour la position de $A_{0} B_{0}$ correspondant à $h^{0}$ * on a $\mathrm{AB}$ au-dessus de $\mathrm{A}^{\prime} \mathrm{B}^{\prime}$ le canal ne peut convenir.

\section{b) Capacité de transport d'un canal.}

La détermination de la capacité de transport maximale s'effectue par un procédé analogue à celui utilisé pour (A) ci-dessus. En posant $Q_{j}^{\prime}=Q_{j}+\Delta$ on obtient une valeur de $h^{0}{ }_{*}(\Delta)$ pour chaque valeur de $\Delta$. On fait varier $\Delta$ jusqu'à ce que $A B$ soit confondu avec $A^{\prime} B^{\prime}$. Les calculs ci-dessus son t notablement simplifiés en substituant $\mathrm{F}(u) / \mathrm{K}_{4}$ à $\mathrm{I}(u)$.

2. Cas $i \neq 0$.

Des tables directement:

$$
f(u)=\frac{0,005}{f_{2}(u)-f_{2}\left(u_{n}\right)}, \quad g(u)=\frac{0,005 f_{1}(u)}{f^{2}(u)-f_{2}\left(u u_{n}\right)}
$$

fonction de $u$ et $u_{n}$; la valeur 0,005 s'explique par le fait que l'intervalle $\Delta u$ des tables est égal à 0,005 .

On a donc directement:

ou :

$$
\begin{aligned}
& x=\frac{1}{\mathbf{K}_{4}}\left[\sum_{u_{0}}^{u} f(u)\right]-\frac{1}{\mathbf{K}_{3}}\left[\sum_{u_{0}}^{u} g(u)\right] \\
& x=\frac{1}{\mathbf{K}_{4}}\left[\sum_{u_{0}}^{u_{n}} f(u)\right]
\end{aligned}
$$

Lorsque $f_{1}(n)$ est très faible devant $\mathrm{K}_{1}$, le fait que cette fonction varie peu conduit souvent à adopter la forme $\left(3^{\prime *}\right)$ :

en prenant:

$$
x=\frac{1}{\mathrm{~K}_{4}^{\prime}}\left[\sum_{u_{0}}^{u_{11}} f(u)\right]
$$

$$
\frac{1}{\mathrm{~K}_{4}^{\prime}}=\frac{1}{\mathrm{~K}_{3}}\left[\mathrm{~K}_{1}-\frac{f_{1}\left(u_{n}\right)+f_{1}\left(u_{0}\right)}{2}\right]
$$

Des tables donnent aussi directement :

et :

$$
f^{\star}(u)=\int_{u_{\mathrm{n}}+\varepsilon}^{u} \frac{d u}{f_{2}(u)-f_{2}\left(u_{n}\right)}
$$

avec :

$$
g^{\star}(u)=\int_{u_{n}+\varepsilon}^{u} \frac{f_{1}(u) d u}{f_{2}(u)-f_{2}\left(u_{n}\right)}
$$

$$
\varepsilon= \pm 0,05
$$

On a évidemment :

ou :

$$
x=\frac{1}{\mathbf{K}_{4}}\left|f^{\star}(u)\right|_{u_{\mathrm{u}}}^{u_{4}}+\frac{1}{\mathrm{~K}_{3}}\left|\mathbf{G}^{\star}(u)\right|_{u^{0}}^{u_{1}}
$$

$$
x=\frac{1}{\mathrm{~K}_{4}}\left|f^{*}(u)\right|_{u^{0}}^{u_{1}}
$$

La formule (7) s'utilise de la même façon que (6) dont elle ne diffère que par l'introduction du terme $\mathrm{K}_{2} \mathrm{R}(u)$.

\section{Cas des canaux en régime uniforme.}

$A_{3}$. - Cas d'une section de force quelconque.

Il est rare qu'on ait l'occasion d'utiliser $(5,1)$ qui, pour un canal de profil donné, fournit une relation entre $Q, l, i, u_{n}$ (donc $h_{n}$ ).

$B_{3}$. - Cas d'une section de rorme trapézordale.

Il est par contre fréquent d'avoir besoin, pour certaines parties de projet, de déterminer le gabarit d'un canal en régime uniforme. On dispose des formules ci-dessous valables pour $\beta_{1}$ et $\beta_{2}$ quelconques. 
1) $\Omega=\frac{l^{2}}{s}\left(u^{2}+u\right)=\frac{l^{2}}{s} \Omega^{\star}(u) ; \mathrm{X}=l(1+m u)$;

$$
u=\frac{s h}{l} ; \quad l=\frac{s h}{u} ; \quad h=\frac{l}{s} u
$$

2) $l=\left(\frac{\mathrm{Q}^{2}}{i \mathrm{C}}\right)^{3 / 16} \mathfrak{f}(u) ; \quad h=\left(\frac{\mathrm{Q}^{2}}{i \mathrm{C}}\right)^{3 / 16} \mathscr{H}(u)$;

avec :

$$
\mathrm{X}=\left(\frac{\mathrm{Q}^{2}}{i \mathrm{C}}\right)^{3 / 16} \mathfrak{P}(u) ; \quad \Omega=\left(\frac{\mathrm{Q}^{2}}{i \mathrm{C}}\right)^{3 / \mathrm{s}} \mathfrak{S}(u)
$$

3)

$\mathscr{f}(u)=\frac{s^{5 / 8}(1+m u)^{1 / 4}}{\left(u^{2}+u\right)^{5 / 8}} ; \quad \mathscr{H}(u)=\frac{u^{3 / 8}(1+m u)^{1 / 4}}{s^{3 / 8}(1+u)^{5 / 8}}$

$\mathscr{S}(u)=\frac{s^{5 / 8}(1+m u)^{\pi / 4}}{\left(u^{2}+u\right)^{5 / 8}} ; \quad \mathfrak{S}(u)=\frac{s^{1 / 4}(1+m u)^{1 / 2}}{\left(u^{2}+u\right)^{1 / 4}}$

Il résulte de $\overline{1}, \overline{2}$ et $\overline{3}$ que la connaissance de deux des six quantités $\left(\mathrm{Q}^{2} / i \mathrm{C}\right), l, h, \mathrm{X}, \Omega, u$ détermine immédiatement les quatre autres.

On dispose d'une série d'échelles domnant $a^{3 / 16}$, $b^{16 / 3}, c^{3 / 8}, d^{8 / 3}$ el les fonctions :

$$
\mathfrak{l}_{m}(u) ; \quad \mathcal{H} \mathcal{U}_{m}(u) ; \mathscr{P}_{m}(u) ; \mathfrak{S}_{m}(u)
$$

valeurs de :

lorsque :

$$
\mathcal{f}(u), \quad \mathcal{H}(u), \quad \mathscr{P}(u), \quad \mathfrak{S}(u)
$$

$$
\beta_{1}=\beta_{2}=\beta(m) \quad \text { et } \quad s=s(\beta(m))=s(m) ;
$$

lorsque :

on a en posant : $\quad s \neq s(m)$

4)

$$
s^{\star}=\frac{s}{s(m)}
$$

$$
\begin{aligned}
& l=\left(\frac{\mathrm{Q}^{2}}{i \mathrm{C}}\right)^{3 / 16} s^{\star 5 / 8} \mathcal{f}_{m}(u) ; \quad h=\left(\frac{\mathrm{Q}^{2}}{i \mathrm{C}}\right)^{3 / 16} s^{\star}-3 / 8 \mathscr{H}_{m}(u) \\
& \mathrm{X}=\left(\frac{\mathrm{Q}^{2}}{i \mathrm{C}}\right)^{3 / 16} s^{\star 5 / 8} \mathfrak{P}_{m}(u) ; \quad \Omega=\left(\frac{\mathrm{Q}^{2}}{i \mathrm{C}}\right)^{3 / 8} s^{\star 1 / 4} \mathfrak{S}(u)
\end{aligned}
$$

Comme généralement les sections sont symétriques on n'a pratiquement à utiliser que les formules $\overline{1}$, $2,3$.

Si l'on pose :

$$
\mathcal{S}(u)=\mathrm{Y}^{2}(u) \quad \text { on a } \quad \mathfrak{P}(u)=\mathrm{Y}^{s}(u)
$$

Par suite, lorsque le coût $\mathcal{C}$ du projet est de la forme :

$$
\mathcal{C}(\Omega, \mathrm{X}) \quad \text { et que } \frac{\partial \mathrm{C}}{\partial \Omega}>0, \frac{\partial \mathrm{C}}{\partial \mathrm{X}}>0
$$

ce qui est fréquemment le cas, on a :

$$
\frac{d \mathrm{C}}{d u}=\mathrm{A}\left(\mathrm{Y}^{r}\right) \mathrm{Y}^{\prime} u
$$

avec $A$ (Y) évidemment positif. Par suite, le coût minimal est obtenu pour :

donc pour (6):

$$
\mathrm{Y}^{\prime}(u)=0
$$

$$
\frac{2 m}{1+m u}=\frac{2 u+1}{u^{2}+u}
$$

relation vérifiée pour $\mathrm{R}=h / 2$ ainsi que le montre immédiatement l'élimination de $l$ entre les expressions de $\Omega /$ X et $h$ fournies par $\overline{1}$.

L'extremum est un minimum.

On voit que la règle $\mathrm{R}=h / 2$ est valable non seulement pour $\beta_{1}$ et $\beta_{2}$ quelconques, mais pour une explicitation beaucoup plus complexe de $\mathcal{C}$ qu'on ne le fait habituellement.

$l^{\star}, h^{*}, \mathrm{X}^{\star}, \Omega^{\star}$ étant les valeurs de $l, h, \mathrm{X}, \Omega$ vérifiant $\mathrm{R}=(h / 2)$ on $\mathrm{a}$ :

avec:

$$
\begin{aligned}
l^{\star} & =\left(\frac{\mathrm{Q}^{2}}{i \mathrm{C}}\right)^{3 / 16} l^{0} ; \quad h^{\star}=\left(\frac{\mathrm{Q}^{2}}{i \mathrm{C}}\right)^{3 / 16} h^{0} \\
\Omega^{\star} & =\left(\frac{\mathrm{Q}^{2}}{i \mathrm{C}}\right)^{3 / 8} \Omega^{0} ; \quad \mathrm{X}^{\star}=\left(\frac{\mathrm{Q}^{2}}{i \mathrm{C}}\right)^{3 / 16} \mathrm{X}^{0}
\end{aligned}
$$

$$
\begin{aligned}
l^{0} & =\frac{4^{5 / 8}(t-s)}{(2 t-s)^{3 / 8}}, \quad h^{0}=2^{1 / 4}(2 t-s)^{-3 / 8} \\
\Omega^{0} & =[4(2 t-s)]^{1 / 4} ; \quad \mathrm{X}^{0}=[4(2 t-s)]^{5 / 8}
\end{aligned}
$$

obtenues en reportant $u^{\star}=1 /(m-2)$, solution de $\bar{b}$ dans $\mathcal{S}(u)$, $\mathscr{H}(u), \Omega(u), \mathrm{X}(u)$.

Des échelles donnent $l^{0}, h^{0}, \Omega^{0}, \mathrm{X}^{0}$ en fonction de $m$, pour $\beta_{1}=\beta_{2}=\beta(m)$ ou de $t$ et $m$ pour $\beta_{1} \neq \beta_{2}$.

Il arrive que l'on explicite le coût $\mathcal{C}$ par une relation :

On a :

$$
\mathcal{C}=\mathcal{C}(\Omega, \mathrm{X}, l, \mathrm{X}-l)
$$

$$
\mathfrak{v}(u)=\frac{\mathfrak{P}(u)}{1+m u}=\frac{Y^{5}(u)}{1+m u}
$$

et le coût $\mathcal{C}$ prend la forme $\mathcal{C}(Y, z)$ avec $z=\mathrm{Y}^{5} / 1+m u$, ce qui permet d'obtenir une relation $\left.\overline{6}^{\prime}\right) \mathcal{C}^{\prime}(u)=0$ donnant le coût minimal.

Lorsque $\mathcal{C}=\mathcal{C}(\Omega, \mathrm{X})$, avec :

$$
\frac{\partial \mathcal{C}}{\partial \Omega}>0, \quad \frac{\partial \mathcal{C}}{\partial \mathrm{X}}>0,
$$

les formules 5 ) donnent immédiatement les valeurs :

$$
l^{\star}, h^{\star}, \mathrm{X}^{\star}, \Omega^{\star}
$$

Dans le cas général :

$$
\mathcal{C}=\mathcal{C}(\Omega, \mathrm{X}, l, \mathrm{X}-\mathrm{l})
$$

la racine $\mathrm{U}_{\mathrm{L}}{ }^{*}$ de $\overline{\mathbf{6}^{\prime}}$ permet de déterminer par l'intermédiaire de $\overline{2}$ les valeurs de $l, h, \mathrm{x}, \Omega$ donnant $\mathcal{C}$ minimal que nous appellerons encore $l^{\star}, h^{\star}, \mathrm{X}^{\star}, \Omega^{\star}$. $\Omega^{\star}$.

En général $l, h, \mathrm{X}, \Omega$ sont bornés inférieurement et supérieurement. Si les valeurs $l^{\star}, h^{\star}, \mathrm{X}^{\star}, \Omega^{\star}$ respectent ses bornes, le problème est résolu. Mais pratiquement il est fort rare qu'il en soit ainsi, aussi nous procédons de la façon suivante:

Les tables donnent $Y^{\prime}(\boldsymbol{u})$ de façon à compléter $\overline{1}, 2$ et $\overline{5}$ par les formules :

$$
\begin{gathered}
\sqrt{\Omega}=\left(\frac{\mathrm{Q}^{2}}{i \mathrm{C}}\right)^{3 / 16} \mathrm{Y}(u) \\
\mathrm{Y}(u)=\sqrt{\mathfrak{S}(u)}=\frac{s^{1 / s}(1+m u)^{1 / 4}}{\left(u^{2}+u\right)^{1 / 8}} \\
\mathrm{Y}^{0}=\sqrt{\Omega^{0}}
\end{gathered}
$$

Prenons le cas général où l'on s'impose une vitesse maximale $V_{\mathrm{M}}$, une vitesse minimale $\mathrm{V}_{m}$ :

$$
l_{m} \leqslant l \leqslant l_{\mathrm{MI}} \quad h_{m} \leqslant h \leqslant h_{\mathrm{MI}} \quad \mathrm{X}_{m} \leqslant \mathrm{X} \leqslant \mathrm{X}_{\mathrm{MI}}
$$

l'indice $m$ désignant le minimum fixé et l'indice $M$ le maximum fixé, les bornes pouvant être éventuellement nulles ou égales à $+\infty$. 
On a :

$$
\sqrt{\Omega_{m}}=\sqrt{\frac{\mathrm{Q}}{\mathrm{V}_{\mathrm{XI}}}} \quad \sqrt{\Omega_{\mathrm{M}}}=\sqrt{\frac{\mathrm{Q}}{\mathrm{V}_{m}}}
$$

On calcule :

$$
\begin{gathered}
\mathrm{K}=\left(\frac{i \mathrm{C}}{\mathrm{Q}^{2}}\right)^{3 / 10} ; \mathrm{K} . l_{m} ; \mathrm{K} l_{\mathrm{M}} ; \mathrm{K} h_{m} ; \mathrm{K}_{\mathrm{M}} ; \mathrm{KX}_{m} ; \mathrm{KX}_{\mathrm{M}} \\
a_{m}=\mathrm{K} \sqrt{\frac{\mathrm{Q}}{\mathrm{V}_{\mathrm{M}}}} \quad a_{\mathrm{M}}=\mathrm{K} \sqrt{\frac{\mathrm{Q}}{\mathrm{V}_{m}}}
\end{gathered}
$$

Comme $\mathcal{f}(u)$ est une fonction décroissante de $u$, $\mathscr{H}(u)$ une fonction croissante de $u$,

$$
\mathrm{X}(u), \mathcal{S}(u) \text { et } \mathrm{Y}(u)
$$

des fonctions décroissantes pour $0 \leqslant u<u^{*}$ et croissantes pour $u>u^{*}$, on a les résultats suivants :

- il faut $\mathrm{K} \sqrt{\left(\mathrm{Q} / \mathrm{V}_{\mathrm{M}}\right)} \geqslant \mathrm{Y}^{0}$ :

$$
u_{l, m}, u_{l, \mathrm{M}}, u_{h_{m}}, u_{h_{\mathrm{M}}}, u_{\mathrm{X}_{m}}, u_{\mathrm{X}_{\mathrm{M}}} u_{q_{m}} \text { et } u_{a_{\mathrm{M}}}
$$

étant données par :

$$
\begin{aligned}
\mathrm{K}_{l_{m}} & =\mathcal{P}\left(u_{l, m}\right), \mathrm{K}_{l_{m}}=\mathcal{P}\left(u_{l, \mathrm{M}}\right), \\
\mathrm{K}_{h_{m}} & =\mathscr{H C}\left(u_{h_{m}}\right), \mathrm{K}_{h_{\mathrm{M}}}=\mathcal{H}\left(u_{h_{\mathrm{M}}}\right), \\
\mathrm{K}_{\mathrm{X}_{m}} & =\mathscr{P}\left(u_{\mathrm{X}_{m}}\right), \mathrm{K}_{\mathrm{X}_{\mathrm{M}}}=\mathscr{P}\left(u_{\mathrm{X}_{\mathrm{M}}}\right), \\
a_{m} & =\mathrm{Y}\left(u_{u_{m}}\right), a_{\mathrm{M}}=\mathrm{Y}\left(u_{u_{\mathrm{M}}}\right)
\end{aligned}
$$

on doit avoir :

7)

$$
\begin{gathered}
u_{l, \mathrm{M}} \leqslant u \leqslant u_{l, m} ; u_{h_{u \mathrm{H}}} \leqslant u \leqslant u_{h_{\mathrm{M}}} \\
u \leqslant u_{\mathrm{X}_{m}} ; \quad u \geqslant u_{\mathrm{X}_{\mathrm{M}}} ; \quad u \leqslant u_{a_{m}} u \geqslant u_{a_{M}}
\end{gathered}
$$

sinon le problème est impossible car il y a contradiction entre les données.

Lorsque le coût du projet est de la forme $\mathrm{C}=\mathrm{C}(\Omega, \mathrm{X})$ il y a, parmi l'ensemble des valeurs $u$ vérifiant $\overline{7}$, deux valeurs $u_{1}$ et $u_{2.2}: u_{1} \geqslant u_{u_{1}}$ et le plus proche possible de $u_{a_{1}}$ et $u_{2} \leqslant u_{a_{w_{1}}}$ et le plus proche possible de $\boldsymbol{u}_{a_{m_{m}}}$. On calcule $\mathcal{C}\left(\boldsymbol{u}_{1}\right)$ et $\mathcal{C}\left(\boldsymbol{u}_{2}\right)$. La plus petite de ces deux quantités est le coût minimal possible du projet, compte tenu des contraintes. La valeur $u_{m}$ (égale soit à $u_{1}$, soit à $u_{2}$ ) correspondante fournit par les relations 2 les solutions $l\left(\boldsymbol{u}_{m}\right)$, $h\left(u_{m}\right)$ du problème.

Ce procédé qui évite tout tâtonnement se révèle en pratique rapide surtout lorsqu'on ne borne que la vitesse.

\section{Explication de $X$ et $\Omega$.}

On a :

$d \mathrm{X}_{1}=\frac{t_{1}}{s_{1}} d x_{1} \quad d \mathrm{X}_{2}=\frac{t_{2}}{s_{2}} d x_{2} \quad d y=\frac{d x_{1}}{s_{1}}=\frac{d x_{2}}{s_{2}}$

D'où :

$d \mathrm{X}=d \mathrm{X}_{1}+d \mathrm{X}_{2}=2 t d y, \quad d x=d x_{1}+d x_{2}=2 s d y$

d'où :

$$
\begin{gathered}
d \Omega=-\frac{x}{2 s} d x ; \quad d \mathrm{X}=\frac{t}{s} d x \\
\Omega_{0}=\int_{0}^{u_{10}} \frac{x d x}{2 s(x)}, \quad \mathrm{X}_{0}=\int_{0}^{\iota_{0}} \frac{t(x)}{s(x)} d x
\end{gathered}
$$

oll encore :

$$
\Omega_{0}=\sum_{j=0}^{j=0} \frac{x_{j}\left(x_{j+1}-x_{j}\right)}{2 s_{j}}, \quad \mathrm{X}_{0}=\sum_{j=0}^{j-j_{n}} \frac{t_{j}}{s_{j}}\left(x_{j+1}-x_{j}\right)
$$

\section{V. - Conclusion}

Les utilisateurs que nous avons consultés considèrent que les procédés ci-dessus exposés se révèlent commodes et rapides, ce qui est le seul critère intervenant dans tout problème de pur calcul numérique. Il n'y a rien d'autre à ajouter, les avis pouvant varier à l'infini en pareille matière. Il est évident que pour des sections de forme très compliquées le procédé perd son intérêt et nous utilisons alors l'excellente méthode de silber.

1. Abaques réalisés ou en cours de réalisation :

- Abaques permettant le calcul des coefficients $K_{1}$, $\mathrm{K}_{2}, \mathrm{~K}_{3}$;

- Echelles donnant par lecture directe les valeurs de :

$$
a^{3 / 16}, \quad b^{16 / 3}, \quad c^{3 / 8}, \quad d^{8 / 3}, \quad s^{13 / 3}
$$

\section{Tables numériques réalisées.}

Pour $\|$ variant de 0 à 5 avec un pas moyen de 0,01 :

\begin{tabular}{|c|c|c|c|}
\hline FONCTION & $\mathrm{M}^{\prime} / \mathrm{U}$ & 0,105 & 0,110 \\
\hline F 1 & & $0,775 \mathrm{E}+03$ & $0,670 \mathrm{E}+03$ \\
\hline F 2 & 2.400 & $0,177 \mathrm{E}+04$ & $0,151 E+04$ \\
\hline F 2 & 2.600 & $0,181 \mathrm{E}+04$ & $0,155 E+04$ \\
\hline F 2 & 2.800 & $0,185 \mathrm{E}+04$ & $0,158 \mathrm{E}+04$ \\
\hline $\mathrm{F} 2$ & 3.000 & $0,189 E+04$ & $0,162 E+04$ \\
\hline
\end{tabular}

- calcul des fonctions $F_{1}(u), F_{2}(u), F(u), G(u)$, $\mathrm{R}(u)\left({ }^{*}\right)$;

- calcul des fonctions :

et :

$$
\mathrm{F}^{*}(u)=\int_{u_{u}+\varepsilon}^{u} \frac{d u}{\mathrm{~F}_{2}(u)-\mathrm{F}_{2}\left(\mathrm{u}_{n}\right)}
$$

$$
G^{*}(u)=\int_{u_{n}+\varepsilon}^{* u} \frac{\mathrm{F}_{1}(u) d u}{\mathrm{~F}_{2}(u)-\mathrm{F}_{2}\left(u_{n}\right)}
$$

avec

$$
\varepsilon= \pm 0,05
$$

- calcul des fonctions :

$$
\mathcal{L}(u), \quad \mathscr{H}(u), \quad \mathscr{P}(u), \quad \mathfrak{S}(u)
$$

\section{Extraits des tables}

1. Fonction $F_{1}$ et $F_{i g}$.

(*) La calculatrice ne pent imprimel les symboles $f_{1}(u)$ et $f_{0}(u), f^{*}(u)$ et $g^{*}(u)$, par suite de cet impératif on leur substituera dan's la rédaction des tables dont les extraits suivent des symboles respectifs $\mathrm{F}_{1}(u)$ et $\mathrm{F}_{2}(u)$, $\mathrm{F}^{*}(u)$ et $\mathrm{G}^{*}(z)$. Les indices 1 et 2 enlèvent toute ambiguité les symboles $f$ et $g$ figureront dans l'expression définitive ultérieure. 
LA HOUILLE BLANCHE/N 6-1968

Abaque 1

$Q(m 3 / s)$

$K^{\prime} 1=\frac{g l^{5}}{Q^{2}}$
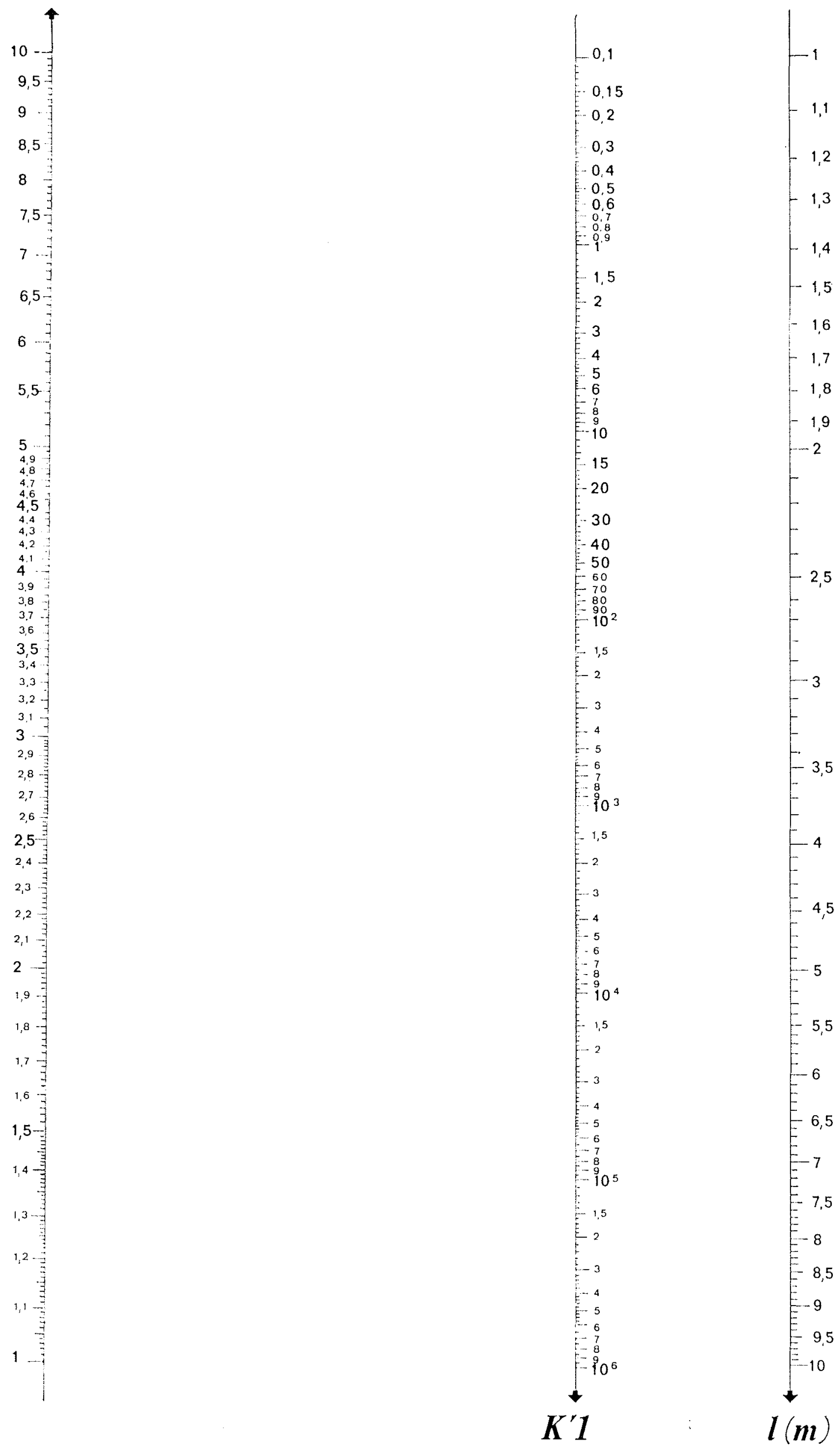
J. NOUgaro, Y. LABYE et G. FUND

$K_{2} K_{\text {, }}$

Abaque 2
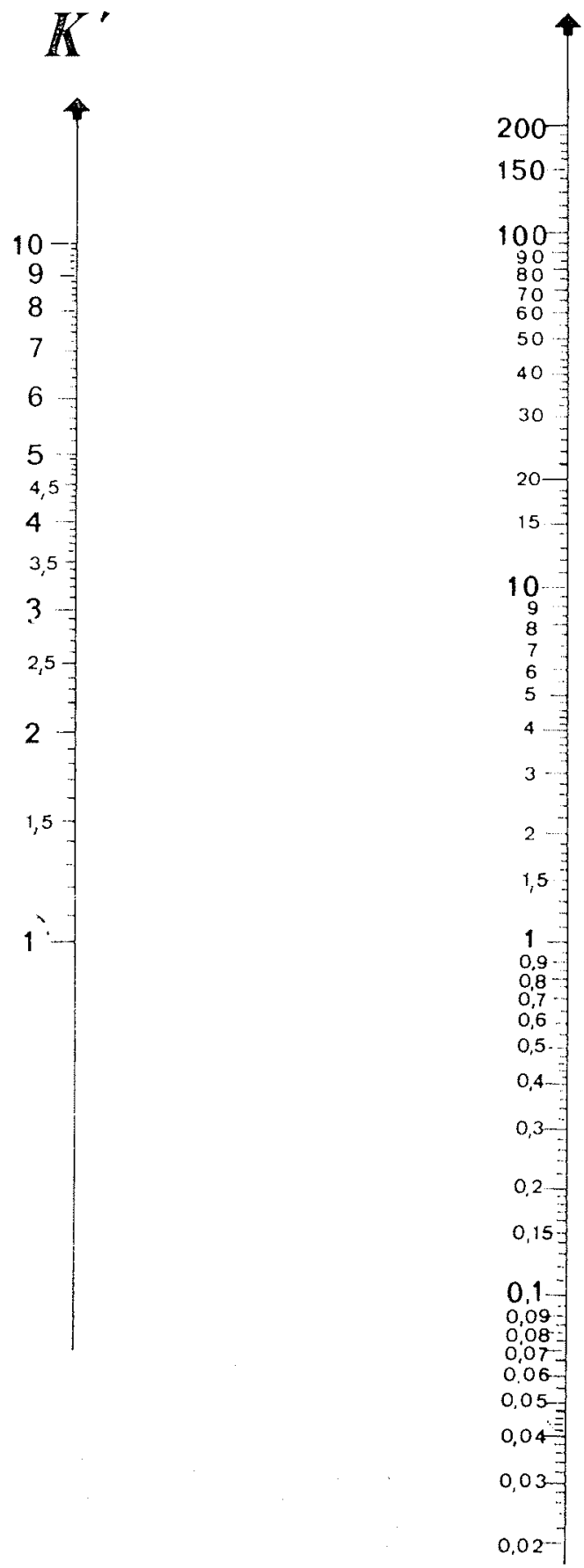
2. Fonction $F$.

\begin{tabular}{|c|c|c|c|}
\hline Fonction & $\mathrm{N}^{\prime} / \mathrm{U}$ & $0,10 \overline{5}$ & 0,110 \\
\hline F & 2.400 & $0,136 \mathrm{E}-04$ & $0,167 \mathrm{E}-04$ \\
\hline $\mathrm{F}$ & 2.600 & $0,134 \mathrm{E}-04$ & $0,163 \mathrm{E}-04$ \\
\hline $\mathrm{F}$ & 2.800 & $0,131 \mathrm{E}-04$ & $0,160 \mathrm{E}-04$ \\
\hline $\mathrm{F}$ & 3.000 & $0,129 \mathrm{E}-04$ & $0,157 \mathrm{E}-04$ \\
\hline
\end{tabular}

\section{Fonction G.}

\begin{tabular}{|c|c|c|c|}
\hline Fonction & $\mathrm{H}^{\prime} / \mathrm{U}$ & 0,105 & 0,110 \\
\hline G & 2.400 & $0,353 E-01$ & $0,375 \mathrm{E}-01$ \\
\hline G & 2.600 & $0,348 \mathrm{E}-01$ & $0,369 \mathrm{E}-01$ \\
\hline G & 2.800 & $0,343 \mathrm{E}-01$ & $0,364 \mathrm{E}-01$ \\
\hline G & 3.000 & $0,339 \mathrm{E}-01$ & $0,359 \mathrm{E}-01$ \\
\hline
\end{tabular}

\section{Fonction $\mathbf{R}$.}

\begin{tabular}{|c|c|c|c|}
\hline Fonction & $M^{\prime} / U$ & 0,105 & 0,110 \\
\hline $\mathrm{R}$ & 2.400 & $0,897 \mathrm{E}-07$ & $0,114 \mathrm{E}-06$ \\
\hline $\mathrm{R}$ & 2.600 & $0,849 \mathrm{E}-07$ & $0,108 \mathrm{E}-06$ \\
\hline $\mathrm{R}$ & 2.800 & $0,805 \mathrm{E}-07$ & $0,102 E-06$ \\
\hline $\mathbf{R}$ & 3.000 & $0,764 \mathrm{E}-07$ & $0,976 \mathrm{E}-07$ \\
\hline
\end{tabular}

\section{- Premier exemple:}

On considère un canal en terre :

$$
k=\frac{1}{\mathrm{C}}=6,25 \times 10^{-4}
$$

dont la pente est $i=1,80 \times 10^{-4}$, la largeur $l=9 \mathrm{~m}$, le débit $\mathrm{Q}=14,8 \mathrm{~m}^{3} / \mathrm{s}$ et la pente des ber ges $p=1 / 2$ (soit $s=2$ ). On établit un barrage tel que le point de départ $A$ est défini par $h_{0}=2,25 \mathrm{~m}$. Déterminer la courbe de remous :

$$
\begin{gathered}
u_{0}=\frac{s h_{0}}{l}=\frac{2 \times 2,25}{9}=0,500 \\
u_{1}=\frac{2 \times 1,80}{9}=0,400
\end{gathered}
$$

Détermination des coefficients au moyen des abaques.

$1^{\circ} K_{1}$ : il s'agit de prendre une valeur de $Q$ comprise entre 0 et 10 .

$$
\begin{aligned}
& \mathrm{K}^{\prime}=\frac{g \times l^{5}}{\mathrm{Q}^{2}} \times \frac{1}{10^{2}} \\
& \mathrm{~K}^{\prime}{ }_{1}=2,8 \times 10^{5} \times 10^{-2}=2,8 \times 10^{3} \\
& \mathrm{~K}_{1}=0,34 \times 10^{3}
\end{aligned}
$$

\begin{tabular}{|c|c|c|c|c|c|c|c|c|c|}
\hline & & \multicolumn{6}{|c|}{ Courbes de remous } & \multicolumn{2}{|c|}{$\Delta u=5.10^{-3}$} \\
\hline$u$ & $\mathbf{F}_{1}(u)$ & $\mathrm{F}_{2}(u)$ & $\mathrm{K}_{1}-\mathrm{F}_{1}(u)$ & $K_{2}-F_{2}(u)$ & $\frac{K_{1}-F_{1}(u)}{F_{2}(u)-K_{2}}$ & $\frac{u}{K_{3}}$ & $x$ & $h$ & $\begin{array}{l}\text { Distaxce } \\
\text { CumuLite }\end{array}$ \\
\hline 0,500 & 4,74 & 7,10 & 335,3 & 9,1 & 36,8 & 6,1 & 224,5 & & \multirow{2}{*}{$\begin{array}{c}0 \\
224,5\end{array}$} \\
\hline 0,495 & 4,91 & 7,37 & 335,1 & 8,83 & 38 & - & 231,8 & 2,23 & \\
\hline \multirow{2}{*}{0,485} & 5,09 & 7,65 & 334,9 & 8,55 & 39,2 & - & 239,1 & \multirow{2}{*}{$\begin{array}{l}2,183 \\
2,16\end{array}$} & 456,3 \\
\hline & 5,27 & 7,95 & 334,7 & 8,25 & 40,6 & - & 247,7 & & $\begin{array}{l}095,11 \\
943,1\end{array}$ \\
\hline \multicolumn{10}{|c|}{ (SUITE DU CALCUL) } \\
\hline 0,450 & 一 & 10 & 333,9 & 6,20 & 53,77 & - & 328 & \multirow{2}{*}{2,025} & 2684,1 \\
\hline \multicolumn{9}{|c|}{ (SUite DU CALCUL) } & \\
\hline \multirow{3}{*}{$\begin{array}{l}0,405 \\
0,400\end{array}$} & 9,42 & 14,8 & 330,6 & 1,40 & 236,14 & - & 1440 & \multirow{3}{*}{$\begin{array}{l}1,823 \\
1,80\end{array}$} & \multirow{3}{*}{$\begin{array}{c}8680,9 \\
11557,9 \\
\infty\end{array}$} \\
\hline & \multirow{2}{*}{9,82} & 15,5 & 330,2 & 0,70 & 471,71 & - & 2877 & & \\
\hline & & 16,2 & & & & & $\infty$ & & \\
\hline
\end{tabular}

$2^{\circ} K_{3}:$

$$
\begin{aligned}
& \mathrm{K}_{3}^{\prime}=\frac{g \times k}{l^{4 / 3}}=3,3 \times 10^{-4} \\
& \mathrm{~K}_{3}=8,2 \times 10^{-4}
\end{aligned}
$$

Les tables nous donnent la valeur de $\mathrm{K}_{2}$ :

$$
\mathrm{K}_{2}=\mathrm{F}_{2}\left(u_{1}\right)=16,2
$$

$$
\mathrm{K}_{2}=16,2
$$




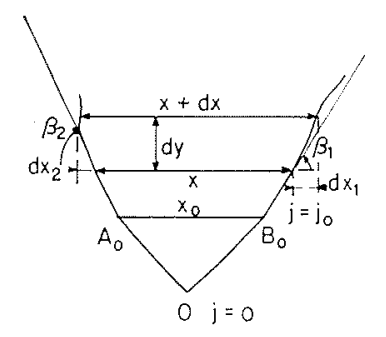

$12 /$

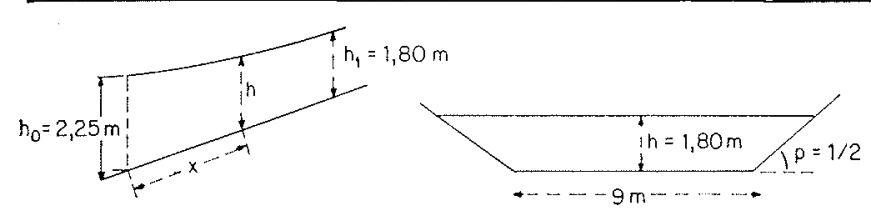

$13 /$

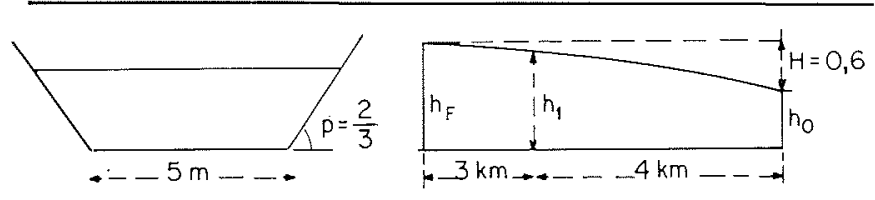

$14 /$

\section{- Deuxileme exempte :}

Déterminer la profondeur d'un canal à fond plat tel que la dénivellation de la surface libre entre l'aval et l'amont soit $\mathrm{H}=60 \mathrm{~cm}$. On a $l=5 \mathrm{~m}$, $p=(2 / 3)(p=$ pente des berges).Le canal comprend deux troncons. Le premier, long de $4 \mathrm{~km}$, a un débit de $5 \mathrm{~m}^{3} / \mathrm{s}$, le second troncon, long de $3 \mathrm{~km}$, a un débit de $3 \mathrm{~m}^{3} / \mathrm{s}$. On a :

$$
\frac{1}{k}=\mathrm{C}=1,600 \times 10^{3}
$$

Appliquons la formule simplifiée :

$$
s^{13 / 3} \sum \frac{\mathrm{Q}^{2} i^{2} k \mathrm{~L}_{i}}{l^{19 / 3}}=\mathrm{F}\left(\mathrm{U}_{0}+\frac{s \mathrm{H}}{l}\right)-\mathrm{F}\left(\mathrm{U}_{0}\right)
$$

L'abaque permettant le calcul de $\mathrm{K}_{2}^{\prime}$ nous donne la valeur de:

$$
\mathrm{A}=\frac{l^{16 / 3}}{k \mathrm{Q}^{2}}
$$

La formule s'écrit done :

$$
\frac{s^{13 / 3}}{l} \Sigma \frac{\mathrm{L}_{i}}{\mathrm{~A}_{i}}=\mathrm{F}\left(\mathrm{U}_{0}+\frac{s \mathrm{H}}{l}\right)-\mathrm{F}\left(\mathrm{U}_{0}\right)
$$

$$
\begin{gathered}
\frac{s^{13 / 3}}{l} \Sigma \frac{\mathrm{L}_{i}}{\mathrm{~A}_{i}}=1,72 \times 10^{-2} \\
\frac{s \mathrm{H}}{l}=0,18 \\
\mathrm{~F}\left(\mathrm{U}_{0}+0,18\right)-\mathrm{F}\left(\mathrm{U}_{0}\right)=1,72 \times 10^{-2}
\end{gathered}
$$

On se sert de la table donnant le calcul de $F(U)$ et on opère par approximations successives.

On trouve $\mathrm{U}_{0}=0,36$, si $h_{0}$ est la profondeur aval :

$$
\mathrm{U}_{0}=\frac{s \times h_{0}}{l}=>\underline{h_{0}}=\frac{0,36 \times 5}{1,5}=\underline{1,20} \mathrm{~m}
$$

Si $h_{f}$ est la profondeur amont, on a :

$$
\underline{h_{f}=1,20 \times 0,6=1,80 \mathrm{~m}}
$$

\section{- Trorsitime Exemple:}

Déterminer la hauteur d'eau dans un canal trapézoïdal en régime uniforme ayant les caractéristiques suivantes :

$$
\begin{aligned}
\mathrm{Q} & =7,70 \mathrm{~m}^{3} / \mathrm{s} \\
\mathrm{I} & =1,50 \times 10^{-4} \\
p \text { (pente des berges) } & =2, \text { soit } s=\frac{1}{2} \\
\mathrm{C} & =\frac{1}{k}=1,600 \times 10^{3} \\
l & =8 \mathrm{~m}
\end{aligned}
$$

A partir des données, il est possible de calculer fo (li) :

$$
\mathcal{f}(u)=l \times\left(\frac{i \times \mathrm{C}}{\mathrm{Q}^{2}}\right)^{3 / 16 i}=2,845
$$

La table nous donne la valeur de $u$ correspondant à la valeur de $\mathcal{L}(u)$ trouvée, soit :

Par suite :

$$
u=0,1
$$

$$
\begin{aligned}
& u=\frac{s \times h}{l}=>h=\frac{l \times u}{s} \\
& h=\frac{8 \times 01}{0,5}=\frac{0,8}{0,5}=1,60 \mathrm{~m}
\end{aligned}
$$

On pourrait de la même façon calculer les autres caractéristiques dı canal. 


\section{Discussion}

M. le Président remercie M. LABYE et constate que la méthode présentée a un caractère très général. Il demande s'il est possible de se procurer les abaques ou les tables qui ont ciû être établis pour faciliter son application, car les résultats des calculs développés sont d'un grand intérêt.

M. LABYE signale que des tables sont en cours de préparation et seront publiees.

M. Carlen remarque que la méthode de calcul exposée semble s'appliquer uniquement aux régimes permanents, lo débit restant le mème dans toutes les sections transversales. Peut-on, le cas echéant, tenir compte des apports ou des prélèvements de débit le long du profil?

Par ailleurs, s'agissant, en somme, d'une méthode de calcul de la ligne d'eau, M. Carlere aimerait savoir si elle peut s'appliquer aux courbes de remous de diverses catégories (régime fluvial, régime torrentiel, régime critique).

M. Labye précise que le procédé de calcul présenté ne s'applique qu'aux débits permanents dans le temps, mais qu'il est valable si le débit varie le long du canal. Il explique :

«Dans le cas où l'on envisage les canaux à fond plat, l'expression :

$$
\Sigma Q_{i}^{2} \mathrm{~L}_{i}=I(n, l)
$$

permet de faire varier les débits sans qu'il en résulte la moindre complication des calculs. Au contraire, lorsqu'on prend l'équation sous sa forme différentielle, on obtient une explicitation particulière d'équation de courbe de remous. On est alors obligé de travailler section par section, puisque les paramètres qui interviennent dans les constantes $K_{i}$ sont fonction du débit.

La formule est valable quel que soit le type d'écoulement où l'on se tronve; ce qui peut varier, du fait même de cette généralité, ce sont les signes de

$$
\begin{array}{ll}
\text { et de } & f_{: 1}(u)-f_{2}\left(u_{u}\right) \\
& \mathrm{K}_{1}-f_{1}(\mathrm{u})
\end{array}
$$

Ceci permet de calculer rapidement les hauteurs normales : $h_{n}$ critiques : $h$, , les pentes critiques : $i^{\prime}$ relative a $Q, i_{e}$ relative à $h ; u, u_{n}, u_{:}$étant les valeurs de $u$ relatives lespectivement a $h, h_{n}, h_{c}$, on obtient en posant :

$$
f_{2}(n)=\Psi\left[f_{1}(n)\right]
$$

Ies relations suivantes:

$$
\begin{gathered}
f_{:}\left(u_{n}\right)=\mathrm{K}_{2} \quad f_{1}\left(u_{c}\right)=\mathrm{K}_{1} \quad i_{c}^{\prime}=\frac{\Psi\left(\mathrm{K}_{1}\right)}{\mathrm{K}^{*}{ }_{2}} \\
i_{c}=\frac{f_{2}(u)}{\mathrm{K}^{*}:} \quad \frac{i_{c}}{i^{\prime}{ }_{c}}=\frac{f_{2}(u)}{\Psi\left(\mathrm{K}_{1}\right)}
\end{gathered}
$$

avec :

$$
\mathrm{K}_{2}^{*}=\mathrm{K}_{2,}(i=1)=\frac{\mathrm{C}^{\prime} 6^{2} l^{p^{10 / 3} / \mathrm{s}}}{\mathrm{s}^{\prime 10 / 3} \mathrm{Q}_{2}}
$$

En outre, Iorsque $i$ est différent de zéro on à :

$$
\begin{gathered}
i_{n}^{\prime}=\frac{i \Psi\left(\mathrm{K}_{1}\right)}{\mathrm{K}_{2}} \quad i_{c}=\frac{i f_{2}(u)}{\mathrm{K}_{2}} \\
i_{c}=\frac{i f_{2}(u)}{\mathrm{K}_{2}}=\frac{i f_{2}(u)}{f_{2}\left(u_{n}\right)}
\end{gathered}
$$

Chaque methode constitue une expression de la même equation différentielle qui, selon son auteur, facilite le calcul numérique $\gg$.

M. RTMÉninas demande quels sont les avantages et les inconvénients de la méthode proposée par M. LABYE par rapport a celle de M. Sirrers, tontes deux étant des méthodes de calcul numérique.

\section{LABYE répond :}

«Lorsque le polygone délimitant la section n'a qu'un nombre peu élevé de côtés, cette méthode me paraît beaucoup plus rapide que celle de Silber. Au contraire, quand on a affaire à une section de forme compliquée, la méthode de Silber me semble nettement preferable. Les sections s'explicilent rapidement avec mon procédé sous réserve qu'elles ne soient pas trop «compliquées», de facon à ne pas être oblige d'opérer de nombreux changements suceessifs de variables. D'autre part, celui qui dispose d'un petit calculateur de poche peut faire des programmes très simples permettant de calculer les constantes et les variables; c'est ce qui demande le plus de temps lorsqu'on travaille « mamuellement. $\gg$

Soulignons pour terminer que le calcul des courbes de remous ne constitue qu'une partie de l'admirable traité de Silber qui permet de résoudre de nombreux autres problèmes d'hydraulique à l'aide de methodes particulièrement élégantes».

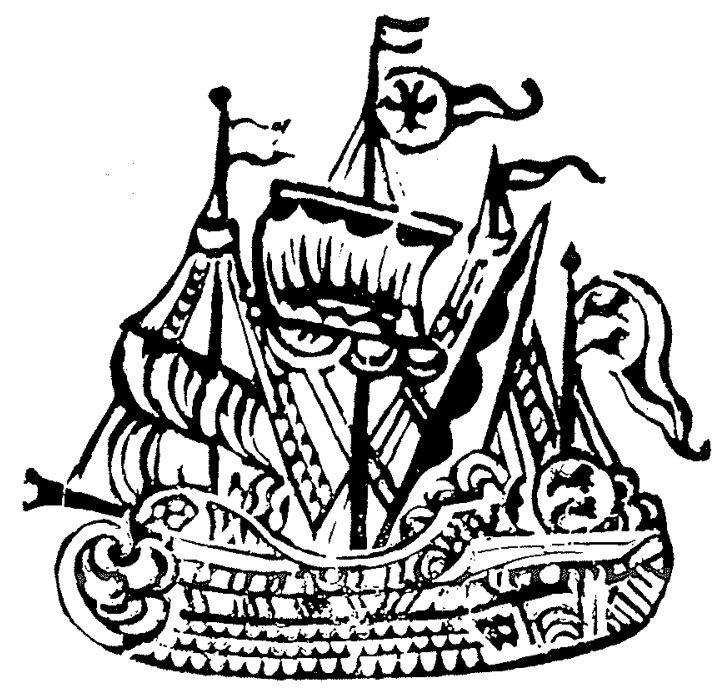

Gravure du XVII siècle 
rappelle

ses volumes

hors-série

et ses

numéros

spéciaux

\section{HYDRAULIQUE ET ELECTRICITE FRANÇAISES}

Edition anglaise : Un volume (1950), 413 pages............. Broché

\section{$\boldsymbol{B} O \boldsymbol{R} \mathbf{T}$}

Un volume (1953), 367 pages.

Broché

\section{DONZERE-MONDRAGON}

Un volume (1955), 486 pages. Relié

\section{TIGN $\mathbf{I S}$}

Un volume $(1958), 440$ pages....................... Relié

\section{LE DANUBE}

No 2/1964 de “ La Houille Blanche ", 218 pages

\section{LES ENERGIES DE LA MER}

IV ${ }^{e s}$ Journées de l'Hydraulique de la Société Hydrotechnique de France (1956). Deux volumes, 751 pages........................ Brochés

\section{TURBINES ET POMPES HYDRAULIQUES}

$V^{e s}$ Journées de l'Hydraulique de la Société Hydrotechnique de France (1958). Deux volumes, 730 pages....................... Brochés

\section{L'HYDRAULIQUE SOUTERRAINE}

Vles Journées de l'Hydraulique de la Société Hydrotechnique de France (1960). Deux volumes, 663 pages.......................... Brochés

\section{BULLES ET GOUTTES}

La tension superficielle en hydraulique VIIIs Journées de I'Hydraulique de la Société Hydrotechnique de France (1962). Deux volumes, 624 pages............................. Brochés

\section{LES INSTABILITES EN HYDRAULIQUE} ET EN MECANIQUE DES FLUIDES

VIII'es Journées de l'Hydraulique de la Société Hydrotechnique de France (1964). Deux volumes, 674 pages.......................... Brochés

\section{L'HYDRAULIQUE DU POINT DE VUE MEDICAL} № 2/1966 de "La Houille Blanche ", 150 pages........ Broché $\left\{\begin{array}{r}\text { France : } 25 \mathrm{~F} \\ \text { Etranger: }\end{array}\right.$

MACHINES HYDRAULIQUES : ETUDES RÉCENTES No 2-3/1968 de «La Houille Blanche », 150 pages.............. Broché 